Article

\title{
Time-Fractional Diffusion-Wave Equation with Mass Absorption in a Sphere under Harmonic Impact
}

\author{
Bohdan Datsko ${ }^{1,2}$, Igor Podlubny ${ }^{3}$ and Yuriy Povstenko ${ }^{4, *}$ \\ 1 Faculty of Mathematics and Applied Physics, Rzeszow University of Technology, Powstancow Warszawy 8, \\ 35-959 Rzeszow, Poland; datskob@prz.edu.pl \\ 2 Institute for Applied Problems of Mechanics and Mathematics NAS of Ukraine, 79060 Lviv, Ukraine \\ 3 BERG Faculty, Technical University of Kosice, B. Nemcovej 3, 04200 Kosice, Slovakia; igor.podlubny@tuke.sk \\ 4 Faculty of Mathematical and Natural Sciences, Jan Dlugosz University in Czestochowa, \\ Armii Krajowej 13/15, 42-200 Czestochowa, Poland \\ * Correspondence: j.povstenko@ajd.czest.pl; Tel.: +48-343-612-269
}

Received: 24 April 2019; Accepted: 12 May 2019; Published: 16 May 2019

\begin{abstract}
The time-fractional diffusion equation with mass absorption in a sphere is considered under harmonic impact on the surface of a sphere. The Caputo time-fractional derivative is used. The Laplace transform with respect to time and the finite sin-Fourier transform with respect to the spatial coordinate are employed. A graphical representation of the obtained analytical solution for different sets of the parameters including the order of fractional derivative is given.
\end{abstract}

Keywords: fractional calculus; mass absorption; diffusion-wave equation; Caputo derivative; harmonic impact; Laplace transform; Fourier transform; Mittag-Leffler function

\section{Introduction}

The classical parabolic diffusion equation with heat or mass absorption [1]

$$
\frac{\partial u}{\partial t}=a \Delta u-b u
$$

also describes bioheat transfer, lateral surface mass or heat exchange in a thin plate, heating of tissue during laser treatment irradiation, etc. (see, for example [2-5]). The Klein-Gordon equation

$$
\frac{\partial^{2} u}{\partial t^{2}}=a \Delta u-b u
$$

is used in solid state physics, classical mechanics, nonlinear optics, and quantum field theory [6,7].

The time-fractional equation

$$
\frac{\partial^{\alpha} u}{\partial t^{\alpha}}=a \Delta u-b u, \quad 0<\alpha \leq 2,
$$

can be considered as the extension of the parabolic Equation (1) and hyperbolic Equation (2) and was studied in several publications [8-14].

It should be noted that such a generalization of many classical differential equations with integer derivatives has numerous applications in rheology, geology, physics, plasma physics, chemistry, geophysics, engineering, biology, bio-engineering, finance, and medicine (see [15-29], among many others). There is a great variety of inhomogeneous media where transport phenomena exhibit anomalous properties, the investigation of which is essential to refine understanding the basic characteristics of complex systems widely met in nature. Therefore, studying fractional equations 
has generated increasing attention of scientists in many disciplines. At present, the fractional diffusion-wave equation is generally used to describe a large class of systems at different scales (from the molecular [30] to the space one [31]) which cover media of the diverse nature (from plasma physics [29] to living tissue [3]). The study of this equation is also of interest from the point of view of understanding the complex spatio-temporal dynamics in nonlinear systems of fractional order [32,33].

In Equation (3) and further in this paper, for more concise notation, $\frac{\mathrm{d}^{\alpha} f(t)}{\mathrm{d} t^{\alpha}}$ denotes the Caputo fractional derivative $[16,34]$

$$
\frac{\mathrm{d}^{\alpha} f(t)}{\mathrm{d} t^{\alpha}}=\frac{1}{\Gamma(n-\alpha)} \int_{0}^{t}(t-\tau)^{n-\alpha-1} \frac{\mathrm{d}^{n} f(\tau)}{\mathrm{d} \tau^{n}} \mathrm{~d} \tau, \quad n-1<\alpha<n,
$$

and $\Gamma(\alpha)$ denotes the gamma function.

Angström was the first to investigate the standard parabolic heat conduction equation under harmonic impact and laid the foundations for the new area of study known as "oscillatory diffusion" or "diffusion-waves" (see [35-37] and references therein). Periodic solutions of the bioheat equation were investigated in [38]. The harmonic point source in the bioheat equation was used in therapeutic hypotermia [39,40]; applications of the time-harmonic impact in ultrasound surgery were studied in [41].

As a rule, in the previous studies of diffusion or heat conduction equation the quasi-steady-state oscillations were investigated when the solution $u(\mathbf{x}, t)$ was represented as a product of a function of the spatial coordinates $U(\mathbf{x})$ and the time-harmonic term $\mathrm{e}^{i \omega t}$ with the angular frequency $\omega$

$$
u(\mathbf{x}, t)=U(\mathbf{x}) \mathrm{e}^{i \omega t}
$$

without consideration of the initial conditions.

The use of assumption (5) is based on the well known formula for the derivative of the integer order $n$ of the exponential function

$$
\frac{\mathrm{d}^{n} \mathrm{e}^{\lambda t}}{\mathrm{~d} t^{n}}=\lambda^{n} \mathrm{e}^{\lambda t}
$$

In the event of the non-integer order of time derivative, the assumption (5) cannot be used since [42]

$$
\frac{\mathrm{d}^{\alpha} \mathrm{e}^{\lambda t}}{\mathrm{~d} t^{\alpha}}=\lambda^{\alpha} \mathrm{e}^{\lambda t} \frac{\gamma(n-\alpha), \lambda t)}{\Gamma(n-\alpha)} \neq \lambda^{\alpha} \mathrm{e}^{\lambda t}, \quad n-1<\alpha<n,
$$

with $\gamma(a, x)$ being the incomplete gamma function [43]

$$
\gamma(a, x)=\int_{0}^{x} \mathrm{e}^{-u} u^{a-1} \mathrm{~d} u
$$

It is worthy of notice that for the Riemann-Liouville fractional derivative [16,34] with the lower limit of integration at 0

$$
D_{R L}^{\alpha} f(t)=\frac{\mathrm{d}^{n}}{\mathrm{~d} t^{n}}\left[\frac{1}{\Gamma(n-\alpha)} \int_{0}^{t}(t-\tau)^{n-\alpha-1} f(\tau) \mathrm{d} \tau\right], \quad n-1<\alpha<n,
$$

we also have [16]

$$
D_{R L}^{\alpha} \mathrm{e}^{\lambda t}=t^{-\alpha} E_{1,1-\alpha}(\lambda t) \neq \lambda^{\alpha} \mathrm{e}^{\lambda t}
$$

Here $E_{\alpha, \beta}(z)$ is the Mittag-Leffler function in two parameters $\alpha$ and $\beta[16,34]$

$$
E_{\alpha, \beta}(z)=\sum_{n=0}^{\infty} \frac{z^{n}}{\Gamma(\alpha n+\beta)}, \quad \Re(\alpha)>0, \quad \beta \in C, \quad z \in C .
$$


In this paper, the initial-boundary-value problem for Equation (3) is studied in a spherical domain for the case of central symmetry under the Dirichlet boundary condition varying harmonically in time. The present paper develops and extends the results of the previous investigations [44,45], where the corresponding problems for line and half-line domains were investigated.

\section{Statement of the Problem}

The time-fractional diffusion equation with mass absorption (mass release) is examined in a sphere

$$
\frac{\partial^{\alpha} u}{\partial t^{\alpha}}=a\left(\frac{\partial^{2} u}{\partial r^{2}}+\frac{2}{r} \frac{\partial u}{\partial r}\right)-b u, \quad 0<r<R, \quad 0<t<\infty, \quad 0<\alpha \leq 2
$$

under zero initial conditions

$$
\begin{array}{ll}
t=0: & u=0, \quad 0<\alpha \leq 2 \\
t=0: & \frac{\partial u}{\partial t}=0, \quad 1<\alpha \leq 2
\end{array}
$$

and harmonic impact on the surface of a sphere

$$
r=R: \quad u=u_{0} \mathrm{e}^{i \omega t}
$$

As in the case of classical diffusion equation (when $\alpha=1$ ) and the wave equation (when $\alpha=2$ ) the boundedness condition at the origin is also adopted:

$$
r=0: u \neq \infty
$$

In what follows, the integral transform technique will be used. Recall the Laplace transform rule for the Caputo derivative

$$
\mathcal{L}\left\{\frac{\mathrm{d}^{\alpha} f(t)}{\mathrm{d} t^{\alpha}}\right\}=s^{\alpha} f^{*}(s)-\sum_{k=0}^{n-1} f^{(k)}\left(0^{+}\right) s^{\alpha-1-k}, \quad n-1<\alpha<n,
$$

where the transform is marked by the asterisk, and $s$ is the Laplace transform variable.

The following finite sin-Fourier transform is amenable to the central symmetric problem in a spherical domain $0 \leq r \leq R$ [46]. For the Dirichlet boundary condition:

$$
\begin{gathered}
\mathcal{F}\{f(r)\}=\widetilde{f}\left(\xi_{k}\right)=\int_{0}^{R} r f(r) \frac{\sin \left(r \xi_{k}\right)}{\xi_{k}} \mathrm{~d} r \\
\mathcal{F}^{-1}\left\{\widetilde{f}\left(\xi_{k}\right)\right\}=f(r)=\frac{2}{R} \sum_{k=1}^{\infty} \xi_{k} \widetilde{f}\left(\xi_{k}\right) \frac{\sin \left(r \xi_{k}\right)}{r},
\end{gathered}
$$

where the transform is marked by the tilde, and

$$
\xi_{k}=\frac{k \pi}{R}
$$

is the Fourier transform variable.

For the central symmetric Laplace operator

$$
\mathcal{F}\left\{\frac{\mathrm{d}^{2} f(r)}{\mathrm{d} r^{2}}+\frac{2}{r} \frac{\mathrm{d} f(r)}{\mathrm{d} r}\right\}=-\xi_{k}^{2} \widetilde{f}\left(\xi_{k}\right)+(-1)^{k+1} R f(R) .
$$


Applying to the problems (12)-(16) the Laplace transform with respect to time $t$ and the finite sin-Fourier transform (18) with respect to the radial coordinate $r$, we get in the transform domain

$$
\widetilde{u}^{*}\left(\xi_{k}, s\right)=(-1)^{k+1} a R u_{0} \frac{1}{s^{\alpha}+a \tilde{\xi}_{k}^{2}+b} \frac{1}{s-i \omega} .
$$

The solution is obtained after inversion of the integral transforms:

$$
u(r, t)=\frac{2 a u_{0}}{r} \sum_{k=1}^{\infty}(-1)^{k+1} \xi_{k} \sin \left(r \xi_{k}\right) \int_{0}^{t} \tau^{\alpha-1} E_{\alpha, \alpha}\left[-\left(a \xi_{k}^{2}+b\right) \tau^{\alpha}\right] \mathrm{e}^{i \omega(t-\tau)} \mathrm{d} \tau,
$$

where $E_{\alpha, \beta}(z)$ is the Mittag-Leffler function (11), and the convolution theorem as well as the following equation for the inverse Laplace transform [16]

$$
\mathcal{L}^{-1}\left\{\frac{s^{\alpha-\beta}}{s^{\alpha}+\gamma}\right\}=t^{\beta-1} E_{\alpha, \beta}\left(-\gamma t^{\alpha}\right), \quad \alpha>0, \quad \beta>0,
$$

have been used.

In numerical calculations, the nondimensional quantities are used:

$$
\begin{gathered}
\bar{u}=\frac{u}{u_{0}}, \quad \bar{r}=\frac{r}{R}, \quad \bar{t}=\frac{a^{1 / \alpha}}{R^{2 / \alpha}} t, \quad \bar{\omega}=\frac{R^{2 / \alpha}}{a^{1 / \alpha}} \omega, \\
\bar{b}=\frac{R^{2}}{a} b, \quad \eta_{k}=R \xi_{k}=k \pi .
\end{gathered}
$$

Hence, using in integral in (23) the substitution $\tau=t w$, for the real part of the solution we get

$$
\bar{u}(\bar{r}, \bar{t}, \bar{b}, \bar{\omega})=\frac{2 \bar{t}^{\alpha}}{\bar{r}} \sum_{k=1}^{\infty}(-1)^{k+1} \eta_{k} \sin \left(\bar{r} \eta_{k}\right) \int_{0}^{1} w^{\alpha-1} E_{\alpha, \alpha}\left[-\left(\eta_{k}^{2}+\bar{b}\right) \bar{t}^{\alpha} w^{\alpha}\right] \cos [\bar{\omega} \bar{t}(1-w)] \mathrm{d} w,
$$

where we can see that the solution $\bar{u}$ depends not only on time and spatial coordinate, but also on the parameters $\bar{b}$ and $\bar{\omega}$.

To simplify calculations, it would be worthwhile to introduce a substitution $z=w^{\alpha}$. Hence,

$$
\bar{u}(\bar{r}, \bar{t}, \bar{b}, \bar{\omega})=\frac{2 \bar{t}^{\alpha}}{\bar{r}} \sum_{k=1}^{\infty}(-1)^{k+1} \eta_{k} \sin \left(\bar{r} \eta_{k}\right) \int_{0}^{1} E_{\alpha, \alpha}\left[-\left(\eta_{k}^{2}+\bar{b}\right) \bar{t}^{\alpha} z\right] \cos \left[\bar{\omega} \bar{t}\left(1-z^{1 / \alpha}\right)\right] \mathrm{d} z .
$$

To evaluate the Mittag-Leffler function the algorithms suggested in [47] were used; see also the MATLAB function [48] that implements these algorithms.

The numerical results are shown in Figures 1-5. Calculations were carried out for the grid size step $\Delta \bar{t}=0.1, \Delta \bar{r}=0.05$. A graphical representation of the solution (27) makes it possible to analyze not only the limiting cases of the problem (see Section 3), but also to understand the influence of the main parameters of the problem (including the value of the order of fractional derivative) on the spatial-temporal evolution of the solution.

It is seen from Figures that time oscillations of the solution are governed by the harmonic term $\mathrm{e}^{i \omega t}$. The increasing of absorption parameter $b$ decreases the oscillation amplitude (Figures $2 \mathrm{~b}$ and $3 \mathrm{~d}$ ), whereas increasing $\alpha$ increases it (Figures $2 \mathrm{a}-\mathrm{d}$ and $3 \mathrm{~d}$ ). As the Mittag-Leffler function

$$
E_{2,2}\left(-x^{2}\right)=\frac{\sin x}{x}
$$

the space oscillations of the solution depending on the order of fractional derivative appear for $\alpha \geq 1.5$ (Figures $1 \mathrm{~d}$ and $2 \mathrm{~d}$ ) and become well-marked for $\alpha$ approaching 2 . The influence of both factors is evident from Figures 4 and 5. 


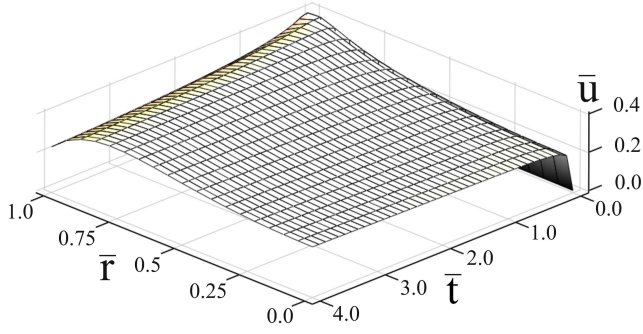

(a)

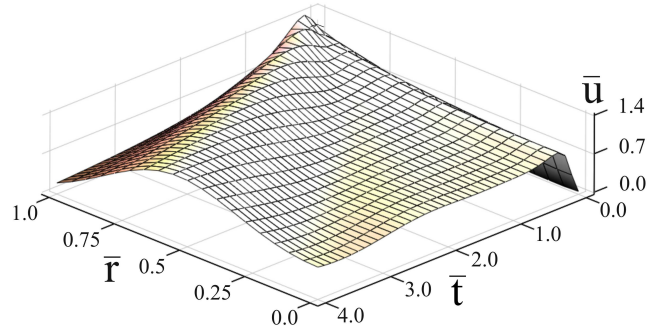

(c)

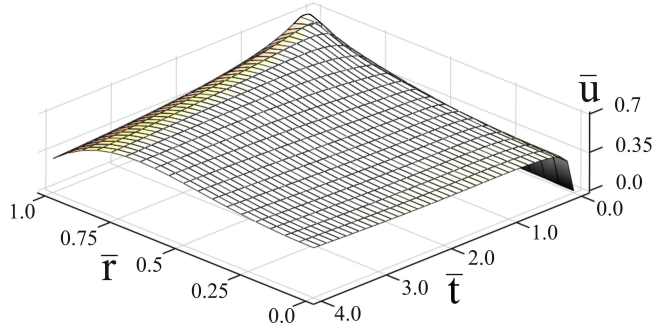

(b)

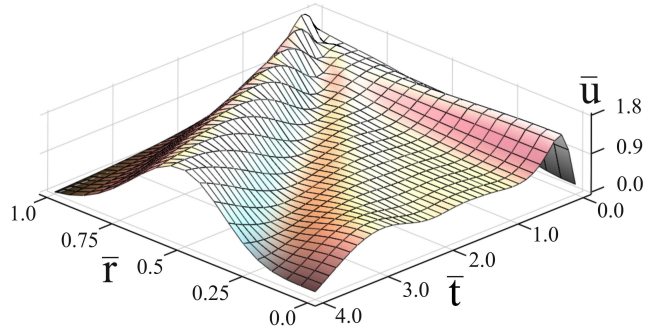

(d)

Figure 1. Evolution of the solution for the problems (12)-(15) under constant impact. The results of computer simulation of the formula (27) for the parameters $\bar{b}=4, \bar{\omega}=0$ and different values of $\alpha$ : $\alpha=0.5-(\mathbf{a}) ; \alpha=0.75-(\mathbf{b}) ; \alpha=1.25-(\mathbf{c}) ; \alpha=1.5-(\mathbf{d})$.

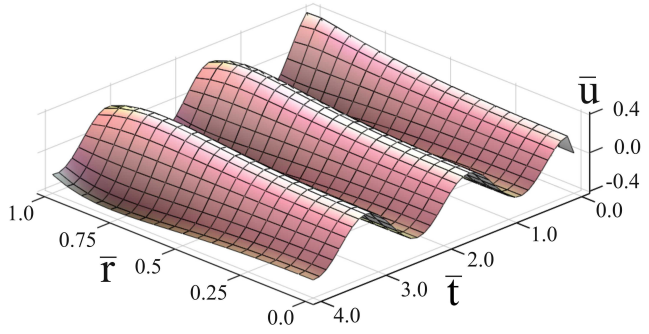

(a)

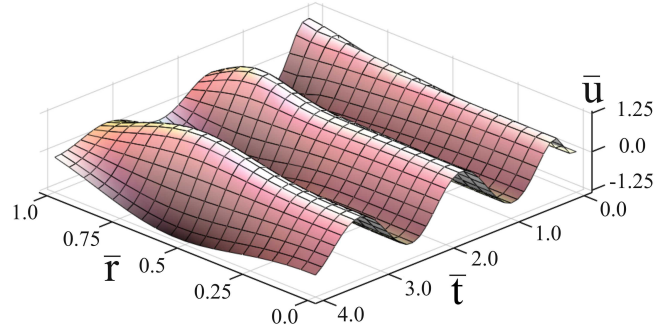

(c)

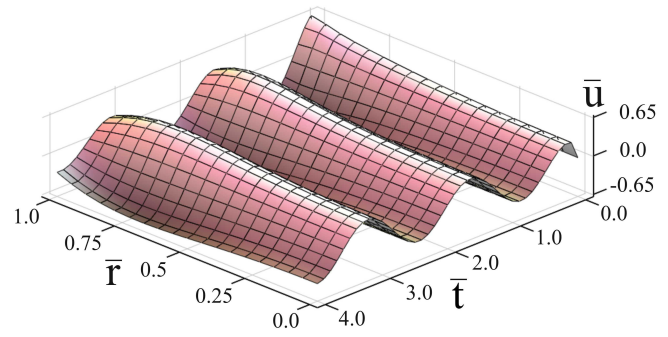

(b)

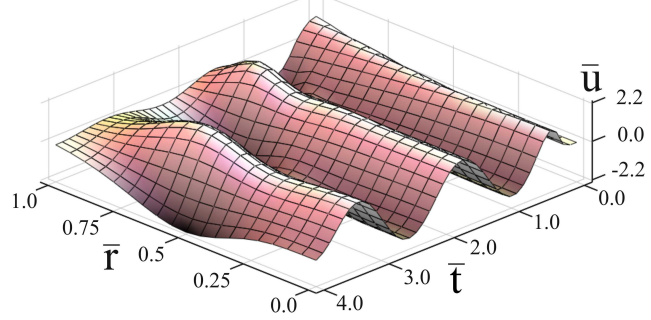

(d)

Figure 2. Evolution of the solution for the problems (12)-(15) under harmonic impact. The results of computer simulation of the formula (27) for the parameters $\bar{b}=4, \bar{\omega}=4$ and different values of $\alpha$ : $\alpha=0.5-(\mathbf{a}) ; \alpha=0.75-(\mathbf{b}) ; \alpha=1.25-(\mathbf{c}) ; \alpha=1.5-(\mathbf{d})$. 


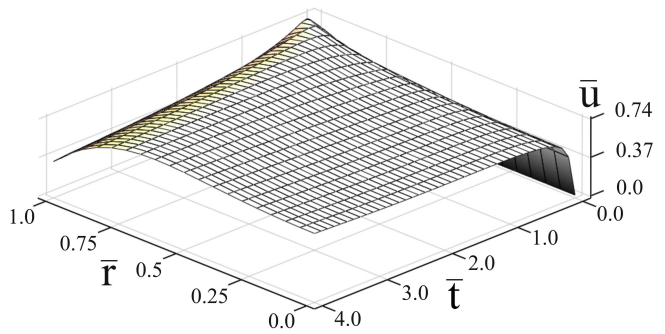

(a)

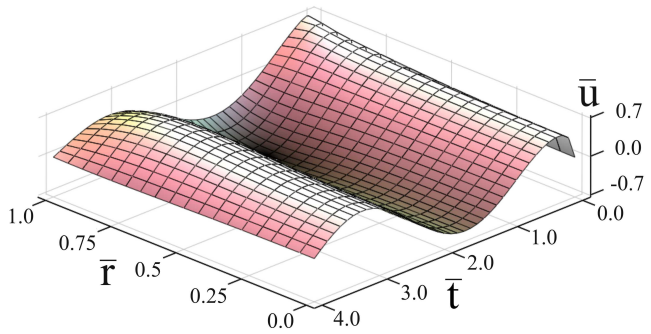

(c)

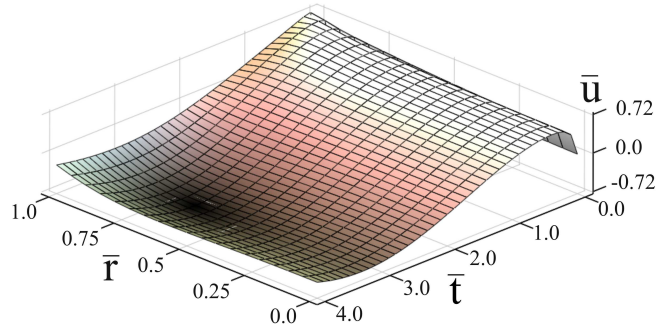

(b)

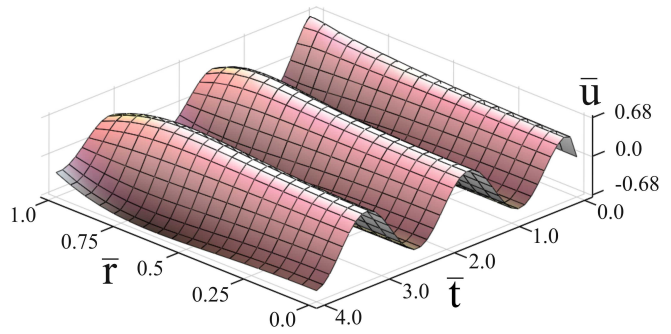

(d)

Figure 3. Evolution of the solution for the problems (12)-(15) with increasing the frequency $\bar{\omega}$ in the sub-diffusive case. The results of computer simulation of the formula (27) for the parameters $\bar{b}=1$, $\alpha=0.75$ and different values of $\bar{\omega}: \bar{\omega}=0.0-(\mathbf{a}) ; \bar{\omega}=1.0-(\mathbf{b}) ; \bar{\omega}=2.0-(\mathbf{c}) ; \bar{\omega}=4.0-(\mathbf{d})$.

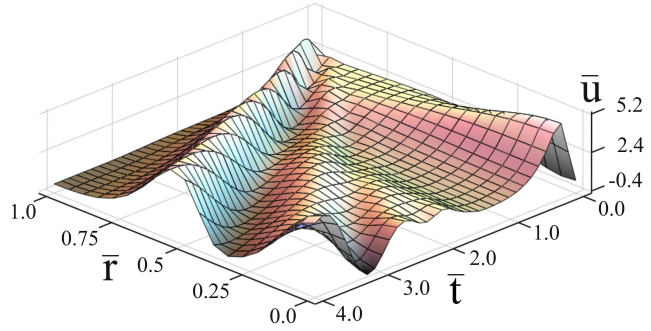

(a)

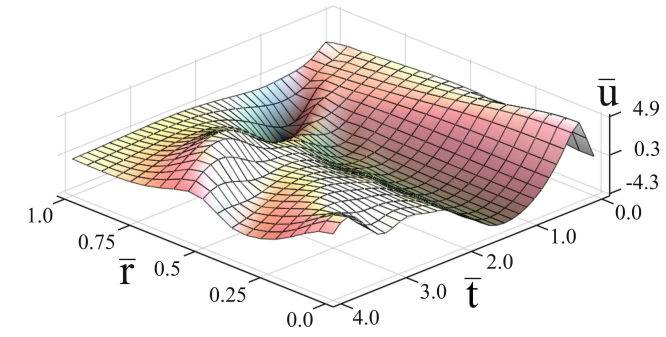

(c)

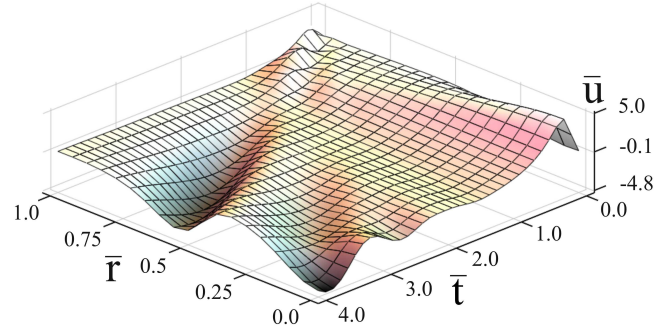

(b)

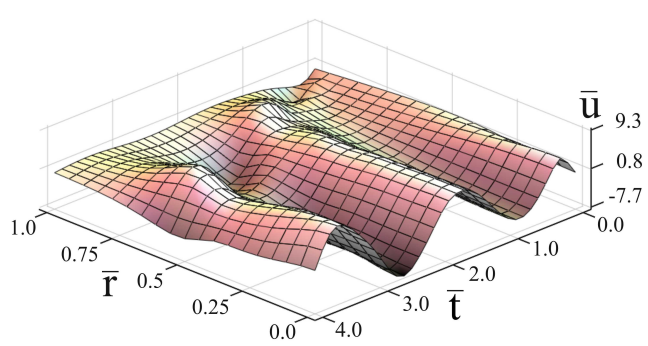

(d)

Figure 4. Evolution of the solution for the problems (12)-(15) with increasing the frequency $\bar{\omega}$ in the sub-wave case. The results of computer simulation of the formula (27) for the parameters $\bar{b}=1$, $\alpha=1.75$ and different values of $\bar{\omega}: \bar{\omega}=0.0-(\mathbf{a}) ; \bar{\omega}=1.0-(\mathbf{b}) ; \bar{\omega}=2.0-(\mathbf{c}) ; \bar{\omega}=4.0-(\mathbf{d})$. 


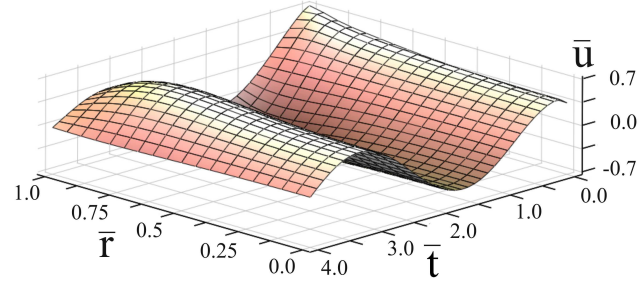

(a)

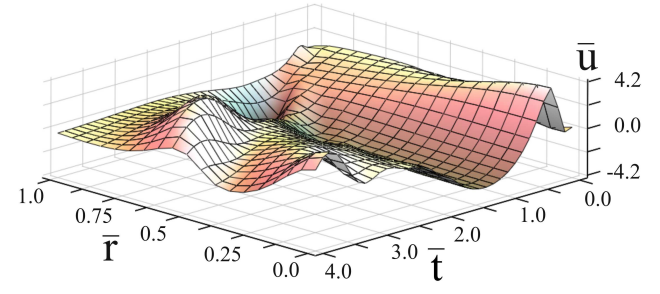

(c)

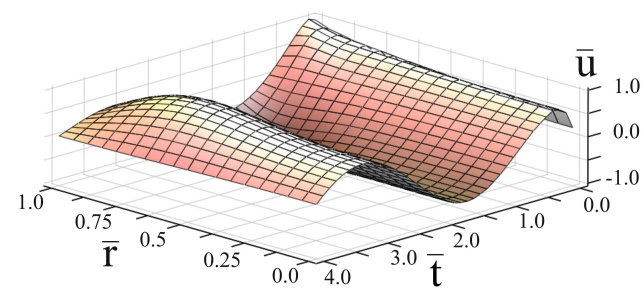

(b)

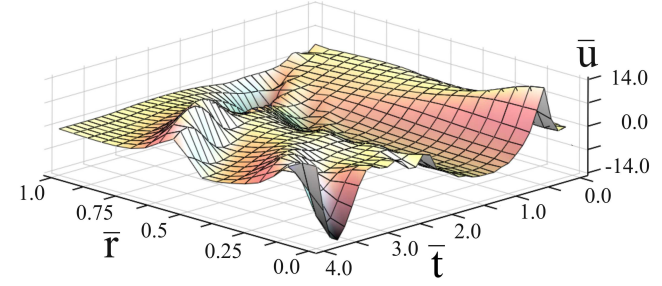

(d)

Figure 5. Evolution of the solution for the problems (12)-(15) for different orders of fractional derivative. The results of computer simulation of the formula (27) for the parameters $\bar{b}=2, \bar{\omega}=2.0$ and different values of $\alpha: \alpha=0.75-(\mathbf{a}) ; \alpha=0.95-(\mathbf{b}) ; \alpha=1.75-(\mathbf{c}) ; \alpha=1.95-(\mathbf{d})$.

\section{Analysis of the Quasi-Steady-State Oscillations}

Now, we shall investigate two particular cases of the problem studied in the previous section corresponding to the integer values of the order of time derivative. For $\alpha=1$, we have

$$
\widetilde{u}^{*}\left(\xi_{k}, s\right)=(-1)^{k+1} a R u_{0} \frac{1}{s+a \tau_{k}^{2}+b} \frac{1}{s-i \omega} .
$$

Taking into account that [49]

$$
\mathcal{L}^{-1}\left\{\frac{1}{(s+p)(s+q)}\right\}=\frac{\mathrm{e}^{-q t}-\mathrm{e}^{-p t}}{p-q},
$$

we arrive at the solution to the bioheat equation

$$
\begin{aligned}
u(r, t) & =\frac{2 a u_{0}}{r} \sum_{k=1}^{\infty}(-1)^{k+1} \frac{\xi_{k} \sin \left(r \xi_{k}\right)}{a \tilde{\xi}_{k}^{2}+b+i \omega} \mathrm{e}^{i \omega t} \\
& -\frac{2 a u_{0}}{r} \sum_{k=1}^{\infty}(-1)^{k+1} \frac{\xi_{k} \sin \left(r \xi_{k}\right)}{a \tilde{\xi}_{k}^{2}+b+i \omega} \mathrm{e}^{-\left(a \tilde{\xi}_{k}^{2}+b\right) t} .
\end{aligned}
$$

Similarly, for $\alpha=2$,

$$
\widetilde{u}^{*}\left(\xi_{k}, s\right)=(-1)^{k+1} a R u_{0} \frac{1}{s^{2}+a \tilde{\xi}_{k}^{2}+b} \frac{1}{s-i \omega}
$$

Taking into consideration that [49]

$$
\mathcal{L}^{-1}\left\{\frac{1}{\left(s^{2}+p^{2}\right)(s+q)}\right\}=\frac{1}{p^{2}+q^{2}}\left[\mathrm{e}^{-q t}-\cos (p t)+\frac{q}{p} \sin (p t)\right],
$$


we obtain the solution to the Klein-Gordon equation

$$
\begin{aligned}
u(r, t) & =\frac{2 a u_{0}}{r} \sum_{k=1}^{\infty}(-1)^{k+1} \frac{\xi_{k} \sin \left(r \xi_{k}\right)}{a \xi_{k}^{2}+b-\omega^{2}} \mathrm{e}^{i \omega t} \\
& -\frac{2 a u_{0}}{r} \sum_{k=1}^{\infty}(-1)^{k+1} \frac{\xi_{k} \sin \left(r \xi_{k}\right)}{a \xi_{k}^{2}+b-\omega^{2}}\left[\cos \left(\sqrt{a \xi_{k}^{2}+b} t\right)+\frac{i \omega}{\sqrt{a \xi_{k}^{2}+b}} \sin \left(\sqrt{a \xi_{k}^{2}+b} t\right)\right] .
\end{aligned}
$$

For integer $\alpha$, we can assume that

$$
u(r, t)=U(r) \mathrm{e}^{i \omega t} .
$$

For $\alpha=1$, the function $U(r)$ fulfills the equation

$$
\frac{\mathrm{d}^{2} U}{\mathrm{~d} r^{2}}+\frac{2}{r} \frac{\mathrm{d} U}{\mathrm{~d} r}-\frac{b+i \omega}{a} U=0,
$$

under the boundary condition

$$
r=R: \quad U(r)=u_{0},
$$

and for $b>0$ has the solution bounded at the origin

$$
U(r)=\frac{R u_{0}}{r} \frac{\sinh [r \sqrt{(b+i \omega) / a}]}{\sinh [R \sqrt{(b+i \omega) / a}]} .
$$

Therefore,

$$
u(r, t)=\frac{R u_{0}}{r} \frac{\sinh [r \sqrt{(b+i \omega) / a}]}{\sinh [R \sqrt{(b+i \omega) / a}]} \mathrm{e}^{i \omega t}
$$

(for negative value of $b$, sinh will be substituted by sin).

The first term in the solution (31) can be evaluated analytically using the following formula [50]

$$
\begin{array}{cl}
\sum_{k=1}^{\infty}(-1)^{k+1} \frac{k}{k^{2}+p^{2}} \sin (k r)=\frac{\pi}{2} \frac{\sinh (r p)}{\sinh (\pi p)}, \quad-\pi<r<\pi, \\
\sum_{k=1}^{\infty}(-1)^{k+1} \frac{k}{k^{2}-p^{2}} \sin (k r)=\frac{\pi}{2} \frac{\sin (r p)}{\sin (\pi p)}, \quad-\pi<r<\pi .
\end{array}
$$

It was emphasized in [51] that Equation (41) is also valid for complex values of $p$ and hence turns into Equation (40) for imaginary $p$.

Taking into account Equation (40), we obtain that the first term in the solution (31) coincides with the quasi-steady-state solution (39), whereas the second term in Equation (31) describes the transient process.

The similar analysis can be carried out for $\alpha=2$ based on the assumption (35). In this case, the function $U(r)$ fulfills the equation

$$
\frac{\mathrm{d}^{2} U}{\mathrm{~d} r^{2}}+\frac{2}{r} \frac{\mathrm{d} U}{\mathrm{~d} r}-\frac{b-\omega^{2}}{a} U=0,
$$

under the boundary condition

$$
r=R: \quad U(r)=u_{0},
$$


and for $b>\omega^{2}$ has the solution bounded at the origin

$$
U(r)=\frac{R u_{0}}{r} \frac{\sinh \left[r \sqrt{\left(b-\omega^{2}\right) / a}\right]}{\sinh \left[R \sqrt{\left(b-\omega^{2}\right) / a}\right]},
$$

whereas for $b<\omega^{2}$

$$
U(r)=\frac{R u_{0}}{r} \frac{\sin \left[r \sqrt{\left(\omega^{2}-b\right) / a}\right]}{\sin \left[R \sqrt{\left(\omega^{2}-b\right) / a}\right]} .
$$

Hence, for $b>\omega^{2}$

$$
u(r, t)=\frac{R u_{0}}{r} \frac{\sinh \left[r \sqrt{\left(b-\omega^{2}\right) / a}\right]}{\sinh \left[R \sqrt{\left(b-\omega^{2}\right) / a}\right]} \mathrm{e}^{i \omega t}
$$

and for $b<\omega^{2}$

$$
u(r, t)=\frac{R u_{0}}{r} \frac{\sin \left[r \sqrt{\left(\omega^{2}-b\right) / a}\right]}{\sin \left[R \sqrt{\left(\omega^{2}-b\right) / a}\right]} \mathrm{e}^{i \omega t} .
$$

The first term in the solution (34) after accounting for Equations (40) and (41) coincides with the quasi-steady-state solutions (46) and (47), respectively, whereas the second term in (34) describes the transient process.

\section{Conclusions}

The time-fractional diffusion-wave equation with the Caputo fractional derivative of the order $0<\alpha \leq 2$ with mass absorption was studied in a spherical domain under the Dirichlet boundary condition varying harmonically in time. The Caputo derivative of the exponential function has a much more complicated form than the corresponding derivative of the integer order. Hence, the assumption that the solution of the problem can be represented as a product of a function of the spatial coordinate and the time-harmonic term without consideration of the initial conditions cannot be used. The solution is obtained using the Laplace transform with respect to time and the finite sin-Fourier transform specifically adapted for a spherical domain and is expressed in terms of the Mittag-Leffler function. A graphical representation of the obtained analytical solution demonstrates the influence of the main parameters of the problem including the value of the order of fractional derivative on the spatial-temporal evolution of the solution.

Author Contributions: All authors have equally contributed to this work. All authors read and approved the final manuscript.

Funding: See acknowledgements of support below. The APC was funded by APVV-14-0892.

Acknowledgments: The first author is thankful for support from Rzeszow University of Technology (grant DS.FD.18.001). The second author acknowledges support provided by grants APVV-14-0892, APVV-18-0526, VEGA 1/0365/19, SK-SRB-18-0011, SK-AT-2017-0015, ARO W911NF-15-1-0228, COST CA15225. The third author would like to acknowledge the support of Jan Dlugosz University in Czestochowa (grant DS/WMP/6011/2018).

Conflicts of Interest: The authors declare no conflict of interest.

\section{References}

1. Crank, J. The Mathematics of Diffusion, 2nd ed.; Clarendon Press: Oxford, UK, 1975.

2. Pennes, H.H. Analysis of tissue and arterial blood temperatures in the resting human forearm. J. Appl. Physiol. 1948, 1, 93-122. [CrossRef] [PubMed]

3. Gafiychuk, V.V.; Lubashevsky I.A.; Datsko, B.Y. Fast heat propagation in living tissue caused by branching artery network. Phys. Rev. E 2005, 72, 051920. [CrossRef] 
4. Datsko, B.Y.; Gafiychuk, V.V.; Lubashevsky, I.A.; Priezzhev, A.V. Self-localization of laser-induced tumor coagulation limited by heat diffusion through active tissue. J. Med. Eng. Technol. 2006, 30, 390-396. [CrossRef]

5. Polyanin, A.D. Handbook of Linear Partial Differential Equations for Engineers and Scientists; Chapman \& Hall/CRC: Boca Raton, FL, USA, 2002.

6. Gravel, P.; Gauthier, C. Classical applications of the Klein-Gordon equation. Am. J. Phys. 2011, 79, 447-453. [CrossRef]

7. Wazwaz, A.-M. Partial Differential Equations and Solitary Waves Theory; Higher Education Press: Beijing, China; Springer: Berlin, Germany, 2009.

8. Abuteen, A.; Freihat, A.; Al-Smadi, M.; Khalil, H.; Khan, R.A. Approximate series solution of nonlinear, fractional Klein-Gordon equations using fractional reduced differential transform method. J. Math. Stat. 2016, 12, 23-33. [CrossRef]

9. Damor, R.S.; Kumar, S.; Shukla, A.K. Solution of fractional bioheat equation in terms of Fox's H-Function. SpringerPlus 2016, 5, 1-10. [CrossRef]

10. Ferrás, L.L.; Ford, N.J.; Morgado, M.L.; Nóbrega, J.M.; Rebelo, M.S. Fractional Pennes' bioheat equation: theoretical and numerical studies. Fract. Calc. Appl. Anal. 2015, 18, 1080-1106. [CrossRef]

11. Golmankhaneh, A.K.; Golmankhaneh, A.K.; Baleanu, D. On nolinear fractional Klein-Gordon equation. Signal Process. 2011, 91, 446-451. [CrossRef]

12. Kheiri, H.; Shahi, S.; Mojaver, A. Analytical solutions for the fractional Klein-Gordon equation. Comput. Meth. Diff. Equ. 2014, 2, 99-114.

13. Qin, Y.; Wu, K. Numerical solution of fractional bioheat equation by quadratic spline collocation method. J. Nonlinear Sci. Appl. 2016, 9, 5061-5072. [CrossRef]

14. Vitali, S.; Castellani, G.; Mainardi, F. Time fractional cable equation and applications in neurophysiology. Chaos Solitons Fractals 2017, 102, 467-472. [CrossRef]

15. Gorenflo, R.; Mainardi, F. Fractional calculus: Integral and differential equations of fractional order. In Fractals and Fractional Calculus in Continuum Mechanics; Springer: Wien, Austria, 1997; pp. 223-276.

16. Podlubny, I. Fractional Differential Equations; Academic Press: San Diego, CA, USA, 1999.

17. Povstenko, Y. Fractional heat conduction equation and associated thermal stresses. J. Therm. Stress. 2005, 28, 83-102. [CrossRef]

18. Magin, R.L. Fractional Calculus in Bioengineering; Begell House Publishers, Inc.: Redding, CA, USA, 2006.

19. Gafiychuk, V.V.; Datsko, B.Y. Spatiotemporal pattern formation in fractional reaction-diffusion systems with indices of different order. Phys. Rev. E 2008, 77, 066210. [CrossRef]

20. Mainardi, F. Fractional Calculus and Waves in Linear Viscoelasticity: An Introduction to Mathematical Models; Imperial College Press: London, UK, 2010.

21. Tarasov, V.E. Fractional Dynamics: Applications of Fractional Calculus to Dynamics of Particles, Fields and Media; Springer: Berlin/Heidelberg, Germany, 2010.

22. Datsko, B.; Luchko, Y.; Gafiychuk, V. Pattern formation in fractional reaction-diffusion systems with multiple homogeneous states. Int. J. Bifurcat. Chaos 2012, 22, 1250087. [CrossRef]

23. Datsko, B.; Gafiychuk, V. Complex nonlinear dynamics in subdiffusive activator-inhibitor systems. Commun. Nonlinear Sci. Numer. Simul. 2012, 17, 1673-1680. [CrossRef]

24. Uchaikin, V.V. Fractional Derivatives for Physicists and Engineers; Springer: Berlin, Germany, 2013.

25. Atanacković, T.M.; Pilipović, S.; Stanković, B.; Zorica, D. Fractional Calculus with Applications in Mechanics: Vibrations and Diffusion Processes; John Wiley \& Sons: Hoboken, NJ, USA, 2014.

26. Herrmann, R. Fractional Calculus: An Introduction for Physicists, 2nd ed.; World Scientific: Singapore, 2014.

27. Povstenko, Y. Fractional Thermoelasticity; Springer: New York, NY, USA, 2015.

28. Datsko, B.; Gafiychuk, V.; Podlubny, I. Solitary travelling auto-waves in fractional reaction-diffusion systems. Commun. Nonlinear Sci. Numer. Simul. 2015, 23, 378-387. [CrossRef]

29. Anderson, J.; Moradi, S.; Rafiq, T. Non-linear Langevin and fractional Fokker-Planck equations for anomalous diffusion by Lévy stable processes. Entropy 2018, 20, 760. [CrossRef]

30. Weiss, M.; Nilsson, T. In a mirror dimly: Tracing the movements of molecules in living cells. Trends Cell Biol. 2004, 14, 267-273. [CrossRef]

31. Zelenyi, L.M.; Milovanov, A.V. Fractal topology and strange kinetics: From percolation theory to problems in cosmic electrodynamics. Phys. Uspekhi 2004, 47, 809-852. [CrossRef] 
32. Gafiychuk, V.; Datsko, B. Different types of instabilities and complex dynamics in reaction-diffusion systems with fractional derivatives. J. Comp. Nonlinear Dyn. 2012, 7, 031001. [CrossRef]

33. Datsko, B.; Gafiychuk, V. Complex spatio-temporal solutions in fractional reaction-diffusion systems near a bifurcation point. Fract. Calc. Appl. Anal. 2018, 21, 237-253. [CrossRef]

34. Kilbas, A.A.; Srivastava, H.M.; Trujillo, J.J. Theory and Applications of Fractional Differential Equations; Elsevier: Amsterdam, The Netherlands, 2006.

35. Mandelis, A. Diffusion waves and their uses. Phys. Today 2000, 53, 29-33. [CrossRef]

36. Mandelis, A. Diffusion-Wave Fields: Mathematical Methods and Green Functions; Springer: New York, NY, USA, 2001.

37. Vrentas, J.S.; Vrentas, C.M. Diffusion and Mass Transfer; CRC Press: Boca Raton, FL, USA, 2013.

38. Lakhssassi, A.; Kengne, E.; Semmaoui, H. Modifed Pennes' equation modelling bio-heat transfer in living tissues: analytical and numerical analysis. Natl. Sci. 2010, 2, 1375-1385. [CrossRef]

39. Kengne, E.; Lakhssassi, A.; Vaillancourt, R. Temperature distributions for regional hypothermia based on nonlinear bioheat equation of Pennes type: Dermis and subcutaneous tissues. Appl. Math. 2012, 3, 217-224. [CrossRef]

40. Fasano, A.; Sequeira, A. Hemomath. The Mathematics of Blood; Springer: Cham, Switzerland, 2017.

41. Malinen, M.; Huttunen, T.; Kaipio, J.P. Thermal dose optimization method for ultrasound surgery. Phys. Med. Biol. 2003, 48, 745-762. [CrossRef]

42. Povstenko, Y. Fractional heat conduction in a space with a source varying harmonically in time and associated thermal stresses. J. Therm. Stress. 2016, 39, 1442-1450. [CrossRef]

43. Abramowitz, M.; Stegun, I.A. (Eds.) Handbook of Mathematical Functions with Formulas, Graphs and Mathematical Tables; Dover: New York, NY, USA, 1972.

44. Povstenko, Y.; Kyrylych, T. Time-fractional diffusion with mass absorption under harmonic impact. Fract. Calc. Appl. Anal. 2018, 21, 118-133. [CrossRef]

45. Povstenko, Y.; Kyrylych, T. Time-fractional diffusion with mass absorption in a half-line domain due to boundary value of concentration varying harmonically in time. Entropy 2018, 19, 346. [CrossRef]

46. Povstenko, Y. Linear Fractional Diffusion-Wave Equation for Scientists and Engineers; Birkhäuser: New York, NY, USA, 2015.

47. Gorenflo, R.; Loutchko, J.; Luchko, Y. Computation of the Mittag-Leffler function and its derivatives. Fract. Calc. Appl. Anal. 2002, 5, 491-518.

48. Podlubny, I. Mittag-Leffler Function; Calculates the Mittag-Leffler Function with Desired Accuracy, MATLAB Central File Exchange, File ID 8738. Available online: www.mathworks.com/matlabcentral/ fileexchange/8738 (accessed on 17 April 2019).

49. Erdélyi, A.; Magnus, W.; Oberhettinger, F.; Tricomi, F. Tables of Integral Transforms; McGraw-Hill: New York, NY, USA, 1954; Volume 1.

50. Prudnikov, A.P.; Brychkov,Y.A.; Marichev, O.I. Integrals and Series, Volume 1: Elementary Functions; Gordon and Breach Science Publishers: Amsterdam, The Netherlands, 1986.

51. Magnus, W.; Oberhettinger, F. Formeln und Sätze für die Speziellen Funkttionen der Mathematischen Physik, 2nd ed.; Springer: Berlin, Germany, 1948.

(C) 2019 by the authors. Licensee MDPI, Basel, Switzerland. This article is an open access article distributed under the terms and conditions of the Creative Commons Attribution (CC BY) license (http:/ / creativecommons.org/licenses/by/4.0/). 